\title{
Métodos alternativos para detecção de betalactamase de espectro estendido em Escherichia coli e Klebsiella pneumoniae
}

\author{
Alternative methods for the detection of extended-spectrum-beta-lactamase in Escherichia coli and Klebsiella \\ pneumoniae
}

Alexsander Costa Martins'; Simone Ulrich Picoli

\section{unitermos}

Betalactamase de

espectro estendido

Resistência bacteriana

Escherichia coli

Klebsiella pneumoniae

\section{resumo}

Introdução: A resistência a antimicrobianos tem aumentado rapidamente nos últimos anos no Brasil e no mundo e, embora exista uma variedade de mecanismos de resistência, destaca-se a produção de betalactamase de espectro estendido (ESBL) como um dos principais. Essas enzimas são mediadas por plasmídios, conferem resistência a vários antimicrobianos betalactâmicos e são inibidas por compostos, como ácido clavulânico, sulbactam e tazobactam. Objetivo: O objetivo deste estudo foi comparar metodologias alternativas à técnica padrão preconizada pelo Clinical and Laboratory Standards Institute (CLSI) para detecção de ESBL. Material e método: Foram realizados testes com 36 isolados (26 de E. coli 10 de K. pneumoniae) mediante disco combinado (CLSI) e técnicas alternativas designadas meio disco (MD) e substituição de discos (SD). Conclusão: As duas metodologias propostas apresentaram resultados satisfatórios com sensibilidade superior a $90 \%$ e custo inferior à técnica de referência (disco combinado), podendo ser utilizadas na pesquisa de ESBL. abstract

Introduction: Antimicrobial resistance has increased apace in Brazil and worldwide in the last years, even though there is a great variety of resistance mechanisms and extended spectrum beta-lactamases (ESBL) is among the main ones. These enzymes are plasmid mediated, which causes resistance to some beta-lactam antimicrobials and are inhibited by compounds such as clavulanic acid, sulbactam and tazobactam. Objective: The objective of this study was to compare alternative methods to the standard ESBL detection technique recommended by the Clinical and Laboratory Standards Institute (CLSI). Material and method: Tests with 36 isolates (26 E. coli and $10 \mathrm{~K}$. pneumoniae) were performed by means of CLSI disk diffusion method and alternative techniques designated as half disk (HD) and disk substitution (SD). Conclusion: Both methods yielded satisfactory results with higher sensitivity (90\%) and lower costs in comparison with the reference one (CLSI disk diffusion method), which corroborates its use in ESBL investigation.

\section{key words}

Extended-spectrum-betalactamase

Bacterial resistance

Escherichia coli

Klebsiella pneumoniae

1. Biomédico.

2. Mestra em Microbiologia; professora adjunta da Universidade Feevale. 


\section{Introdução}

A resistência a antimicrobianos tem aumentado rapidamente nos últimos anos no Brasil e no mundo, gerando a necessidade crescente do conhecimento do perfil de sensibilidade das bactérias que mais frequentemente causam infecções e do modo de disseminação da resistência ${ }^{(24)}$. Entre as bactérias Gram-negativas, responsáveis por grande parte das infecções nosocomiais, a produção de betalactamases constitui um dos principais mecanismos de resistência aos antimicrobianos betalactâmicos(17).

A família Enterobacteriaceae é composta por mais de 30 gêneros e 130 espécies $^{(9)}$ de bacilos Gram-negativos que podem ser responsáveis por diferentes doenças infecciosas, sendo isolados de qualquer amostra clínica ${ }^{(18)}$. Nesse grupo, os gêneros mais frequentemente associados são Escherichia sp. e Klebsiella sp., mas também podem envolver espécies de Citrobacter sp., Enterobacter sp., Morganella sp., Proteus sp., Providencia sp., Salmonella sp. e Serratia sp. ${ }^{(2,6,8,11,13,16,22)}$.

Embora exista grande variedade de mecanismos de resistência aos antimicrobianos betalactâmicos, um dos mais importantes é a produção de betalactamases, que são enzimas capazes de hidrolisar o anel betalactâmico de penicilinas, cefalosporinas e outros antimicrobianos relacionados, tornando-os inativos. Destaca-se entre eles a produção de betalactamase de espectro estendido (ESBL), principalmente em algumas espécies de bactérias Gramnegativas ${ }^{(10)}$. Essas enzimas são mediadas por genes plasmidiais não induzíveis e são inibidas por compostos como ácido clavulânico, sulbactam e tazobactam ${ }^{(23)}$.

O uso excessivo de antimicrobianos em humanos e animais, as infecções hospitalares cruzadas, a cadeia alimentar, o comércio e a migração humana parecem ter contribuído para a recente propagação de ESBLs fora dos hospitais, embora o papel desses fatores seja variável e ligado a determinadas situações epidemiológicas ${ }^{(5)}$.

O laboratório de microbiologia tem um importante papel na detecção de bactérias produtoras de ESBL, pois o rápido diagnóstico é necessário para adequado manejo clínico dos pacientes hospitalizados, visto que há dados indicando a relação dessa enzima com complicações clínicas e maiores taxas de mortalidade ${ }^{(20,23)}$. A demora no diagnóstico leva à permanência do paciente no ambiente hospitalar por tempo prolongado ${ }^{(23)}$, possibilitando disseminação intra e inter-hospitalar dessas enzimas ${ }^{(25)}$ antes da implementação de medidas de controle. Além disso, o paciente pode receber terapia inadequada, levando à falha terapêutica.
Segundo o Clinical and Laboratory Standards Institute $(\mathrm{CLSI})^{(3)}$, a confirmação dessa enzima em isolados de Klebsiella, Escherichia coli e Proteus mirabilis deveria ser feita por disco combinado de cefalosporinas de terceira geração com clavulanato. Desde 2010, esse órgão mudou suas recomendações em relação à pesquisa e à confirmação dessa enzima nos isolados dessas enterobactérias por meio da alteração de pontos de corte interpretativos para as cefalosporinas de amplo espectro.

Contudo, no contexto epidemiológico, reforça-se a relevância da pesquisa laboratorial contínua, dada a capacidade elevada de transmissibilidade da ESBL (via plasmídios). Dessa forma, a busca de técnicas alternativas para sua detecção é igualmente necessária para facilitar o rastreamento dessa betalactamase em isolados clínicos comunitários e/ou nosocomiais.

\section{Objetivo}

Comparar metodologias alternativas ao padrãoouro preconizado pelo CLSI para confirmação de ESBL em Escherichia coli e Klebsiella pneumoniae, visando redução de custos e exequibilidade.

\section{Material e método}

Para a realização dessa pesquisa, foram utilizadas 36 cepas positivas para ESBL (método fenotípico confirmatório recomendado pelo CLSI de discos combinados), sendo 26 cepas de Escherichia coli e 10 de Klebsiella pneumoniae provenientes da bacterioteca do Laboratório de Bacteriologia da Universidade Feevale. Elas estavam armazenadas em caldo glicerinado a $-20^{\circ} \mathrm{C}$. Os testes foram realizados entre setembro e outubro de 2009.

Para a execução dos testes, as cepas congeladas foram repicadas em ágar sangue (MCBarth) e incubadas a $35^{\circ} \mathrm{C} \pm$ $2^{\circ} \mathrm{C}$ por 18 a 24 horas. Posteriormente, foi realizado novo repique em ágar McConkey (MCBarth) e a incubação foi feita em condições adequadas. Com o crescimento secundário, foi preparada uma suspensão correspondente à escala 0.5 de McFarland e semeada em placa de ágar Mueller-Hinton (MCBarth) para a realização dos testes fenotípicos de disco combinado (DC) (CLSI), substituição de discos (SD) e meio disco (MD) (metodologias alternativas).

Para o controle de qualidade, foram utilizadas cepas da American Type Culture Colletion (ATCC ${ }^{\circledR}$ ): Escherichia 
coli ATCC $^{\circledast} 25922$ (ESBL negativa) e Klebsiella pneumoniae ATCC $^{\circledR} 700603$ (ESBL positiva).

\section{Deteç̧ão de ESBL pelo teste padrão CLSI disco combinado}

A detecção dessa enzima foi padronizada segundo o documento Performance Standards For Antimicrobial Susceptibility Testing, $19^{\text {th }}$ Informational Supplement M100-S19, de janeiro de 2009, página 120 (CLSI), para cepas de Escherichia coli, Klebsiella pneumoniae, Klebsiella oxytoca e Proteus mirabilis. Se as três primeiras bactérias anteriormente citadas apresentarem halos de inibição diminuídos para betalactâmicos de amplo espectro (ceftazidima [CAZ] $\leq$ $22 \mathrm{~mm}$; aztreonam $\leq 27 \mathrm{~mm}$; cefotaxima [CTX] $\leq 27 \mathrm{~mm}$; ceftriaxona $\leq 25 \mathrm{~mm}$; cefpodoxima $\leq 17 \mathrm{~mm}$ ), deve-se confirmar a presença de ESBL. Na versão subsequente desse documento (20 Informational Supplement M100-S20), de janeiro de 2010, página 30 (CLSI), ocorreram alterações nos pontos de corte interpretativos para os respectivos betalactâmicos, mas ainda são relevantes a pesquisa e a confirmação da enzima sob a ótica epidemiológica.

A confirmação foi realizada com discos de CAZ $30 \mu \mathrm{g}$, ceftazidima + ácido clavulânico (CCAZ) $30 \mu \mathrm{g} / 10 \mu \mathrm{g}$, CTX $30 \mu \mathrm{g}$ e cefotaxima + ácido clavulânico (CCTX) $30 \mu \mathrm{g} / 10 \mu \mathrm{g}$ (Biorad) (Figura). O resultado foi considerado positivo quando a diferença de medida de halos do antimicrobiano com clavulanato em relação ao mesmo sem o inibidor foi $\geq 5 \mathrm{~mm}$.

\section{Detecção de ESBL pelo teste alternativo substituição de disco}

Esse teste consistiu na colocação de dois discos de amoxicilina + ácido clavulânico (AMC) $20 \mu \mathrm{g} / 10 \mu \mathrm{g}$ (Biorad) na superfície de ágar Müeller-Hinton (MCBarth) em temperatura ambiente. Após 30 minutos, os discos foram retirados com pinça estéril e foram colocados, no mesmo local, discos de CAZ $30 \mu \mathrm{g}$ e CTX $30 \mu \mathrm{g}$ (Biorad) (Figura). Após incubação apropriada, os halos que continham ácido clavulânico foram medidos e comparados com aqueles dos discos contendo apenas as cefalosporinas. Diferença $\geq 5 \mathrm{~mm}$ foi considerada cepa positiva para ESBL.

\section{Detecção de ESBL pelo teste alternativo meio disco}

Para esse teste, utilizaram-se duas metades de discos de antimicrobianos dispostos juntamente na placa, de modo

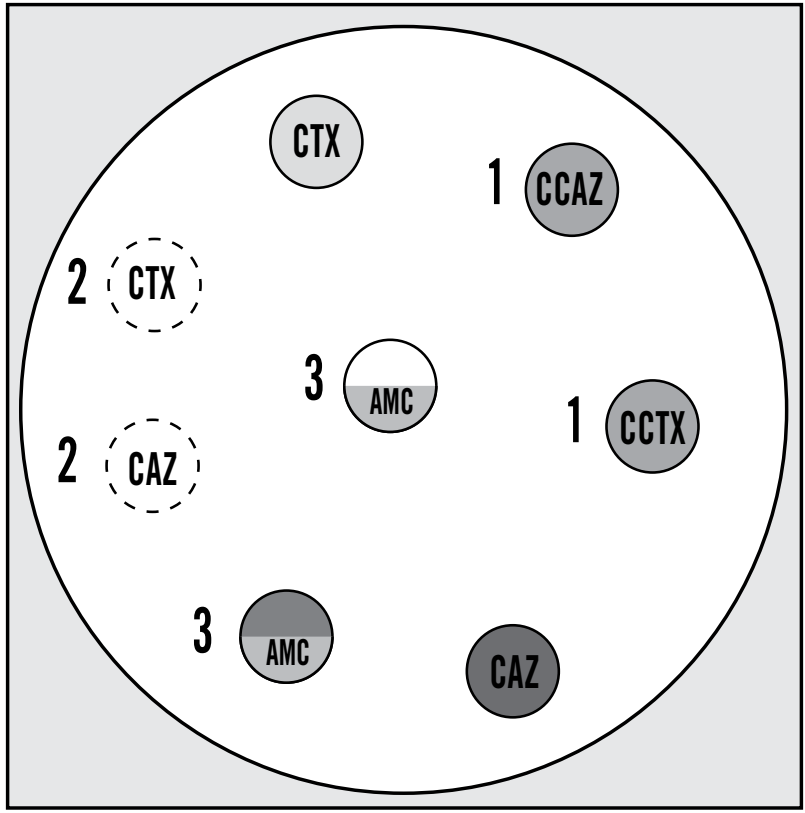

Figura - Representação esquemática da disposição dos discos para os testes de disco combinado (1), substituição de discos (2) e meio disco (3) em placa de ágar Müeller-Hinton (150 mm x $15 \mathrm{~mm}$ )

CCAZ: ceftazidima + ácido clavulânico; CCTX: cefotaxima + ácido clavulânico; CTX: cefotaxima; CAZ: ceftazidima; AMC: amoxicilina + ácido clavulânico.

a formar um único disco (Figura): uma metade de AMC $20 \mathrm{mg} / 10 \mathrm{mg}$ e a outra de CAZ $30 \mathrm{mg}$ ou CTX $30 \mathrm{mg}$ (Biorad). Para a obtenção das metades de discos, cada disco inteiro foi tomado com pinça estéril e cortado ao meio com auxílio de tesoura estéril; as metades de discos foram recolhidas dentro de placa de petri estéril. Os materiais empregados no corte dos discos (pinças e tesouras) foram previamente esterilizados em autoclave $\left(121^{\circ} \mathrm{C}, 15\right.$ minutos). A leitura do teste foi considerada positiva quando a diferença de medida de halos do antimicrobiano com clavulanato em relação ao mesmo sozinho foi $\geq 5 \mathrm{~mm}$ (o diâmetro do halo de inibição foi mensurado na linha de união entre as duas metades dos referidos discos).

\section{Resultados}

Entre os 36 isolados bacterianos produtores de ESBL avaliados, 26 (72,2\%) eram Escherichia coli e 10 (27,8\%) eram Klebsiella pneumoniae. Considerando que uma cepa é dita produtora de ESBL quando o teste é positivo para um ou mais dos substratos empregados, constatou-se que todas as amostras avaliadas apresentaram resultado positivo para a enzima por meio dos testes fenotípicos alternativos $\mathrm{MD}$ e/ou SD. 
O teste padrão de DC, recomendado pelo CLSI, foi positivo nas 36 cepas mediante o substrato CTX, enquanto 26 delas também revelaram ESBL simultaneamente com emprego de CAZ (Tabela).

Nas metodologias alternativas, a técnica MD confirmou ESBL em 34 (94,4\%) cepas, todas detectadas por CTX e $19(52,8 \%)$ delas igualmente positivas diante de CAZ. $\mathrm{O}$ teste SD mostrou resultados semelhantes ao MD: 33 $(91,7 \%)$ cepas positivas mediante CTX e 18 (50\%) também positivas com o substrato CAZ (Tabela). Uma amostra de Escherichia coli foi ESBL positiva somente no teste MD com o substrato $\mathrm{CTX}$.

\section{Discussão}

O trabalho do laboratório de microbiologia é imprescindível na detecção das enterobactérias produtoras de ESBL. A detecção precoce desses microrganismos multirresistentes é muito importante para se instaurarem o tratamento adequado e as medidas de isolamento dos pacientes necessários para se evitar a disseminação desses patógenos ${ }^{(14)}$.

É relevante destacar que a pesquisa fenotípica desse mecanismo enzimático tem relevância tanto no contexto epidemiológico quanto no terapêutico. Após a alteração dos pontos de corte interpretativos para as cefalosporinas e carbapenens (CLSI) $)^{(4)}$ em enterobactérias, iniciou-se uma discussão mundial a respeito dessa mudança. Segundo o documento do CLSI, os novos critérios interpretativos para cefalosporinas já compreendem a presença de mecanismos de resistência como ESBLs e, por essa razão, não seria mais obrigatória a pesquisa confirmatória da referida enzima.

Contudo, diferentes publicações científicas mostram que pode ocorrer falha terapêutica quando infecções por Klebsiella pneumoniae ou Escherichia coli (ESBL positiva), sensível à cefalosporinas de amplo espectro e com baixos valores de concentração inibitória mínima (MIC), são tratadas com esses antibióticos. De acordo com Paterson et al., 40\% das infecções graves causadas por essas bactérias (Escherichia coli ou Klebsiella pneumoniae ESBL positiva com MICs $<2 \mathrm{mg} / \mathrm{l}$ para cefalosporinas de amplo espectro) resultaram em falha no tratamento ${ }^{(19)}$. Por outro lado, Bin et al. relataram êxito terapêutico com emprego de CAZ em infecção severa causada por Escherichia coli, cuja MIC não correspondia à sensibilidade (MIC CAZ 8 mg/l - intermediário). Um aspecto muito importante a ser destacado nessa situação diz respeito à dose de CAZ utilizada: $2 \mathrm{~g}$ a cada 8 horas ${ }^{(1)}$ e não $1 \mathrm{~g}$ a cada 8 horas, como descrito no documento do CLSI 2010.

Algumas publicações que abordam o desfecho de infecções causadas por isolados ESBLs positivos e tratados com cefalosporinas fornecem margem à discussão, pois não indicam a dose de antibiótico utilizada ou não descrevem a MIC para a cefalosporina. Nesse contexto, Kim et al. afirmam que praticamente $43 \%$ dos casos de infecção por bactérias produtoras de ESBL tiveram desfecho não favorável no uso de cefalosporinas, mas não descrevem a MIC correspondente ${ }^{(12)}$, dificultando a interpretação desses resultados.

As taxas de prevalência de ESBL em Escherichia coli e Klebsiella pneumoniae são diversificadas em todo o mundo. Dados do SENTRY Antimicrobial Surveillance Program mostram as variações nesse índice em isolados de Escherichia coli produtores de ESBL: América Latina $=8,5 \%$, Canadá $=4,2 \%, \mathrm{EUA}=3,3 \%$ e Europa $=11,1 \%$; enquanto em Klebsiella pneumoniae os índices nesses mesmos locais são maiores: $45,4 \% ; 4,9 \% ; 7,6 \% ; 22,6 \%$, respectivamente ${ }^{(25)}$.

Dados do SENTRY para o Brasil mostram alta prevalência de isolados produtores de ESBL: 50,3\% em Klebsiella pneumoniae e 9,1\% em Escherichia coli. Embora as taxas de espécies produtoras de ESBL possam variar significativamente de região para região ou mesmo de hospital

Resultados da detecção de ESBL em cepas positivas para a enzima com emprego de metodologias Tabela alternativas (MD e SD)

\begin{tabular}{lccccccc}
\hline Bactéria & $\begin{array}{c}n \\
(\%)\end{array}$ & $\begin{array}{c}\text { DC CAZ } \\
(\%)\end{array}$ & $\begin{array}{c}\text { DC CTX } \\
(\%)\end{array}$ & $\begin{array}{c}\text { MD CAZ } \\
(\%)\end{array}$ & $\begin{array}{c}\text { MD CTX } \\
(\%)\end{array}$ & $\begin{array}{c}\text { SD CAZ } \\
(\%)\end{array}$ & $\begin{array}{c}\text { SD CTX } \\
(\%)\end{array}$ \\
$\begin{array}{l}\text { Escherichia } \\
\text { coli }\end{array}$ & $26(72,2)$ & $20(55,5)$ & $26(72,2)$ & $15(41,7)$ & $24(66,7)$ & $13(36,1)$ & $23(63,9)$ \\
$\begin{array}{l}\text { Klebsiella } \\
\text { pneumoniae }\end{array}$ & $10(27,8)$ & $6(16,7)$ & $10(27,8)$ & $4(11,1)$ & $10(27,7)$ & $5(13,9)$ & $10(27,8)$ \\
Total $(\%)$ & $36(100)$ & $26(72,2)$ & $36(100)$ & $19(52,8)$ & $34(94,4)$ & $18(50)$ & $33(91,7)$ \\
\hline
\end{tabular}

ESBL: betalactamase de espectro estendido; MD: meio disco; SD: substituição de discos; DC: disco combinado; CAZ: ceftazidima; CTX: cefotaxima. 
para hospital em uma mesma região geográfica, as taxas do Brasil são muito superiores àquelas vistas na maioria das outras partes do mundo ${ }^{(21)}$, justificando sua constante pesquisa.

O CLSI recomenda que a triagem e a confirmação de ESBL sejam realizadas para Escherichia coli, Klebsiella pneumoniae, Klebsiella oxytoca e Proteus mirabilis com sensibilidade diminuída diante de betalactâmicos de amplo espectro. Por outro lado, a descrição de ESBL em outras espécies de enterobactérias já foi relatada $(2,6,8,11,13,16,22)$. Contudo, o presente trabalho utilizou somente isolados de Escherichia coli e Klebsiella pneumoniae positivos para ESBL, excluindo, nesse momento, as demais espécies recomendadas.

Nos testes realizados neste estudo, observou-se que grande parte das enzimas foi identificada pelo substrato CTX, sugerindo que a principal enzima ESBL seja uma cefotaximase (CTX-M), corroborando com os dados de outros trabalhos realizados no $\mathrm{Brasil}^{(7)}$ e em outros continentes $^{(15)}$

Ambos os testes alternativos (MD e SD) mostraram sensibilidade superior a $90 \%$, mas o MD $(94,4 \%)$ foi ligeiramente superior ao SD $(91,6 \%)$, denotando a viabilidade de utilizá-los para pesquisa de ESBL.

No tocante à exequibilidade, ficou evidente que ambos os testes necessitam de alguns cuidados em sua confecção: os discos que compõem o MD devem ficar unidos de modo a formar um disco inteiro, enquanto a SD requer a substituição do disco exatamente no local onde o anterior se encontrava. O eventual descumprimento dessas exigências, neste estudo, pode explicar a não confirmação de ESBL em duas cepas por MD e em três por SD. Ainda assim, percebeu-se maior facilidade em executar o teste MD, uma vez que ele não exige cuidados referentes ao tempo para substituição do disco de antimicrobiano.

Em relação aos custos, o valor dos discos necessários para confecção de cada teste foi: $\mathrm{R} \$ 1,68$ no $\mathrm{DC}(\mathrm{CLSI})$, $\mathrm{R} \$ 0,80$ no $M D$ e $R \$ 1,20$ na SD. Pode-se observar que o MD é mais vantajoso financeiramente para o laboratório, pois reduz custos devido ao fato de utilizar somente metade do disco de cada antimicrobiano.

Este estudo apresentou algumas limitações, como a não inclusão das demais cepas produtoras de ESBL descritas no CLSI (Klebsiella oxytoca e Proteus mirabilis) e o número reduzido de amostras avaliadas. Devido à não equivalência de resultados entre as técnicas propostas (MD e SD) em comparação com a metodologia padrão, acredita-se ser prudente não empregá-las em unidades hospitalares com grande incidência de ESBL. De qualquer maneira, fica demonstrada a possibilidade de emprego de técnicas alternativas com boa sensibilidade e menor custo na confirmação desse importante mecanismo de resistência bacteriana, especialmente em locais onde não exista elevada prevalência de ESBL.

\section{Conclusão}

As técnicas alternativas propostas são úteis na confirmação de ESBL em isolados de Escherichia coli e Klebsiella pneumoniae, pois apresentaram sensibilidade satisfatória e custo inferior ao método preconizado pelo CLSI. Assim, ficam indicadas outras metodologias capazes de confirmar esse relevante mecanismo de resistência que dificulta a terapêutica com antibióticos betalactâmicos usualmente empregados na prática clínica.

\section{Referências}

1. BIN, C. et al. Outcome of cephalosporin treatment of bacteremia due to CTX-M-type extended-spectrum beta-lactamase-producing Escherichia coli. Diagn Microbiol Infect Dis, v. 56, n. 4, p. 351-7, 2006.

2. BRADFORD, P. A. Extended-spectrum B-lactamases in the $21^{\text {st }}$ century: characterization, epidemiology, and detection of this important resistance threat. Clin Microb Rev, v. 14, p. 933-51, 2001.

3. CLINICAL AND LABORATORY STANDARD INSTITUTE (CLSI). Performance Standards for Antimicrobial Susceptibility Testing-Table M100-S19. Wayne, PA, USA, 2009.

4. CLINICAL AND LABORATORY STANDARD INSTITUTE (CLSI). Performance Standards for Antimicrobial
Susceptibility Testing-Table M100-S20. Wayne, PA, USA, 2010.

5. COQUE, T. M. et al. Increasing prevalence of ESBLproducing Enterobacteriaceae in Europe. Euro Surveill, v. 13, n. 47, pii=19044, 2008.

6. COUDRON, P. E. et al. Occurrence and detection of expected-spectrum B-lactamases in members of the family Enterobacteriaceae at a Veterans Medical Center: seek and you may find. J Clin Microb, v. 35, p. 2593-7, 1997.

7. D'AZEVEDO, P. A. et al. Laboratory tests in the detection of extended spectrum beta-lactamase production: National Committee for Clinical Laboratory Standards 
(NCCLS) screening test, the E-test, the double disk confirmatory test, and cefoxitin susceptibility testing. Braz J Infect Dis, v. 8, n. 5, p. 372-7, 2004.

8. EMERY, C. L.; WEYMOUTH, L. A. Detection and clinical significance of extended-spectrum B-lactamases in a Tertiary-Care Medical Center. J Clin Microb, v. 35, p. 2061-7, 1997.

9. FARMER III, J. J. et al. Enterobacteriaceae introduction and identification. In: MURRAY, P. R. et al. (Ed.). Manual of clinical microbiology. Washington: ASM Press, 2007. p. 649-69.

10. FRANCISCO, W.; JEA, A. H. Y. Resistência à b-lactamases por presença de ESBL. Disponível em: <www.fleury. com.br/mednews/0301/mdcontfcb0302.htm>. Acesso em: 15 nov. 2009.

11. GANGONUÉ-PIÉBOJI, J. et al. Extended-spectrum-Blactamase-producing Enterobacteriaceae in Yaounde, Cameroon. J Clin Microb, v. 43, p. 3273-7, 2005.

12. KIM, Y. K. et al. Bloodstream infections by extendedspectrum $\beta$-lactamase-producing Escherichia coli and Klebsiella pneumoniae in children: epidemiology and clinical outcome. Antimicrob Agents Chemother, v. 46, n. 5, p. 1481-91, 2002.

13. LUZZARO, F. et al. Trends in production of extendedspectrum B-lactamases among enterobacteria of medical interest: report of the Second Italian Nationwide Survey. J Clin Microb, v. 44, p. 1659-64, 2006.

14. MENEZES, E. A. et al. Freqüência de cepas produtoras de enzima betalactamase de espectro expandido (ESBL) e perfil de susceptibilidade de Klebsiella pneumoniae em hemoculturas no berçário de um hospital de Fortaleza. Rev Bras An Clin, v. 40, n. 1, p. 7-11, 2008.

15. NAVON-VENEZIA, S. et al. Occurrence and phenotypic characteristics of extended-spectrum B-lactamases among members of the family Enterobacteriaceae at the Tel-Aviv Medical Center (Israel) and evaluation of diagnostic tests. J Clin Microb, v. 41, p. 155-58, 2003.

16. NAVON-VENEZIA, S. et al. Plasmid-mediated imipenemhydrolyzing enzyme KPC-2 among multiple carbapenem- resistant Escherichia coli clones in Israel. Antimicrob Agents Chemother, v. 50, p. 3098-101, 2006.

17. NOGUEIRA, K. S. et al. Occurrence of extended-spectrum Beta-lactamases in Enterobacteriaceae isolated from hospitalized patients in Curitiba, southern Brazil. Braz J Infect Dis, v. 10, n. 6, p. 390-5, 2006.

18. O'HARA, C. M. et al. Classification, identification, and clinical significance of Proteus, Providencia and Morganella. Clin Microb Rev, v. 13, p. 534-46, 2000.

19. PATERSON, D. L. et al. Outcome of cephalosporin treatment for serious infections due to apparently susceptible organisms producing extended-spectrum betalactamases: implications for the clinical microbiology laboratory. J Clin Microb, v. 39, n. 6, p. 2206-12, 2001. 20. PITOUT, J. D. D.; LAUPLAND, K. Extendedspectrum B-lactamase-producing Enterobacteriaceae: an emerging public-helth concern. Lancet Infect Dis, p. 159-66, 2008.

21. SADER, H. S. et al. Pathogen frequency and resistance patterns in Brazilian hospitals: summary of results from three years of the SENTRY Antimicrobial Surveillance Program. Braz J Infect Dis, v. 5, p. 200-14, 2001.

22. SPANU, T. et al.; The Italian ESBL Study Group. Occurrence of extended-spectrum B-lactamases in members of the family Enterobacteriaceae in Italy: implications for resistance to $\mathrm{B}$-lactams and other antimicrobial drugs. Antimicrob Agents Chemother, v. 46, p. 196-202, 2002.

23. STÜRENBURG, E.; MACK, D. Extended-spectrum B-lactamases: implications for the clinical microbiology laboratory, therapy, and infection control. J Infect, v. 47, p. 273-95, 2003.

24. TOSIN, I. Avaliação do modo de disseminação da resistência bacteriana a antibacterianos nos hospitais brasileiros. São Paulo: [s. n.], 2001.

25. WINOKUR, P. L. et al. Variations in the prevalence of strains expressing an extended-spectrum B-lactamase phenotype and characterization of isolates from Europe, the Americas, and the Western Pacific Region. Clin Infect Dis, v. 32, suppl. 2, p. S94-103, 2001.

\begin{tabular}{l|l} 
& Endereço para correspondência \\
\hline Simone Ulrich Picoli \\
Universidade Feevale \\
Laboratório de Biomedicina - Instituto de Ciências da Saúde (ICS) \\
RS 239, 2.755 \\
CEP: $93352-000$ - Novo Hamburgo-RS
\end{tabular}

